\title{
Reducción de la actividad física en pacientes con enfermedad pulmonar obstructiva crónica
}

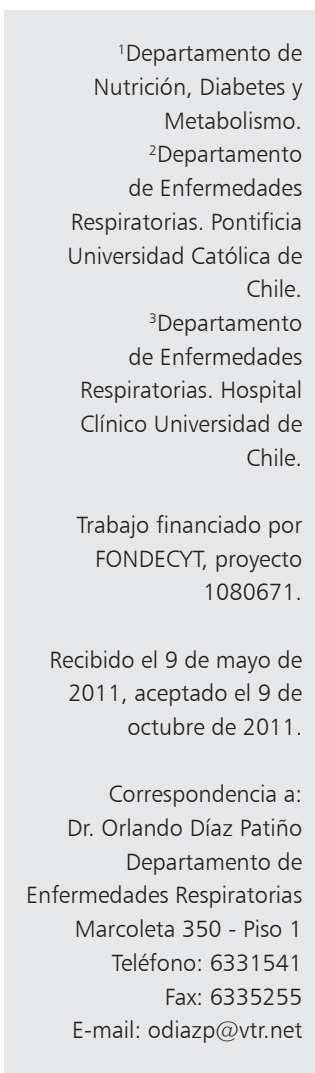

\author{
ALEJANDRA PARADA ${ }^{1}$, JULIETA KLAASSEN ${ }^{1}$, CARMEN LISBOA ${ }^{2}$, \\ FERNANDO SALDÍAS ${ }^{2}$, LAURA MENDOZA ${ }^{3}$, ORLANDO DÍAZ ${ }^{2}$
}

\section{Reduction of physical activity in patients with chronic obstructive pulmonary disease}

Background: Patients with chronic obstructive pulmonary disease (COPD) decrease their physical activity. However, it is unknown at which stage of the disease the reduction occurs and whether dyspnea is a limiting factor. Aim: To compare physical activity between patients with COPD and controls of similar age and to assess its association with disease severity. Material and Methods: We studied 112 patients with mild to very severe COPD and 55 controls. Lung function, six-minutes walking test (SMWT), and physical activity through the International Physical Activity Questionnaire (IPAQ) were measured. Results: Compared to controls, physical activity was significantly reduced in COPD patients (1823 \pm 2598 vs. $2920 \pm 3040$ METs $\mathrm{min} /$ week; $p=0.001$ ). Patients were more frequently sedentary (38 vs. 11\%), while controls were more often very active (31 vs. 19\%) or moderately active (58vs. 43\%). Physical activity was reduced from Global Initiative for Obstructive Chronic Lung Disease (GOLD) stage 2 and from Modified Medical Research Council (MMRC) dyspnea grade 1. Weak relationships were observed between lung function, SMWT and physical activity. Conclusions: Physical activity decreases early in the course of the disease and when dyspnea is still mild, among patients with COPD.

(Rev Med Chile 2011; 139: 1562-1572).

Key words: Motor activity; Pulmonary disease, chronic obstructive; Sedentary lifestyle.
L

a actividad física (AF), definida como cualquier movimiento corporal producido por los músculos esqueléticos que resulta en un gasto energético superior al de reposo ${ }^{1}$, es un parámetro clínico de importancia creciente. Esto se debe a que el nivel de actividad se relaciona con la morbilidad y mortalidad asociada a muchas enfermedades crónicas ${ }^{1}$. Particularmente, en la enfermedad pulmonar obstructiva crónica (EPOC) los niveles bajos de AF se han asociado con la declinación de la función pulmonar ${ }^{2}$, con el número de hospitalizaciones ${ }^{3}$ y con la mortalidad ${ }^{4}$.

La AF puede ser cuantificada empleando diferentes instrumentos. En los pacientes con EPOC ha sido evaluada empleando sensores de movimiento, tales como podómetros y acelerómetros ${ }^{5,6}$; y también utilizando cuestionarios específicos ${ }^{5,7}$. Aunque los sensores de movimiento son más precisos, los cuestionarios son reproducibles, más simples y de menor costo ${ }^{5}$. Existen varios cuestionarios disponibles ${ }^{8}$, entre los cuales el Cuestionario Internacional de Actividad Física (IPAQ; International Physical Activity Questionnaire), posee la ventaja de haber confirmado su confiabilidad y validez en 12 países $^{9}$ y haber incluido países de habla hispana, por lo que existe una versión disponible en español.

Debido a que los síntomas principales en la EPOC son la disnea y fatiga de las extremidades, que conducen paulatinamente a la discapacidad, 
la inactividad es una consecuencia natural de la progresión de la enfermedad. A su vez, la inactividad contribuye a un mayor deterioro físico y a mayor disnea, configurando un círculo vicioso. Por lo tanto, las intervenciones que promuevan la actividad diaria poseen el potencial de romper este círculo vicioso, produciendo mejorías clínicamente relevantes. Por las razones mencionadas, la evaluación de la AF en los pacientes con EPOC ha ganado creciente interés en los últimos años ${ }^{10}$.

Existe escasa información respecto al momento, en la evolución de la EPOC, en que la AF comienza a deteriorarse significativamente. Esto es válido para dos de los índices clínicos más empleados para describir la gravedad de la enfermedad, como son las etapas propuestas por GOLD (Global Initiative for Obstructive Chronic Lung Disease) y la magnitud de la disnea según la escala modificada del Medical Research Council (MMRC) ${ }^{11}$. En consideración a lo anterior, el propósito del presente trabajo ha sido comparar el grado e intensidad de la actividad física realizada por pacientes con EPOC y controles de edad semejante y establecer las posibles asociaciones entre la AF y la gravedad de la enfermedad, de acuerdo a la definición de GOLD y a la magnitud de la disnea según la escala del MMRC.

\section{Material y Método}

\section{Sujetos}

Pacientes. Se estudiaron 112 pacientes con EPOC durante la fase estable de la enfermedad, esto es, al menos ocho semanas desde la última exacerbación. El diagnóstico y la etapa de la enfermedad se establecieron siguiendo las definiciones de GOLD ${ }^{12}$. Los criterios de exclusión fueron: a) antecedentes de asma; b) enfermedades pulmonares concomitantes; y c) otras enfermedades que pudiesen afectar la AF, específicamente enfermedades neurológicas con déficit motor significativo; enfermedades renales en diálisis; enfermedades cardiovasculares sintomáticas, a pesar de tratamiento adecuado; cardiopatía isquémica sintomática o que estuviese recibiendo sólo tratamiento médico; enfermedades neuromusculares; y enfermedades del aparato locomotor.

Controles. El grupo de sujetos sin enfermedad pulmonar estuvo constituido por 55 individuos, voluntarios de nuestra institución, sujetos que consultaron en el policlínico en quienes se descartó enfermedad pulmonar, y vecinos de los pacientes, invitados a participar por vía telefónica. Los criterios de exclusión fueron similares a los de los pacientes.

Todos los participantes fueron citados en dos oportunidades en el lapso de una semana, entre los meses de diciembre de 2010 y marzo de 2011, con el fin de evitar influencias estacionales sobre la actividad física ${ }^{13}$. En la primera visita se recolectaron datos socio-demográficos, magnitud de la disnea, y se efectuaron las pruebas de función pulmonar y de ejercicio. En la segunda visita los participantes respondieron el cuestionario de actividad física. El estudio fue aprobado por el Comité de Ética de la Institución y antes del reclutamiento los pacientes y los controles firmaron un consentimiento informado.

\section{Mediciones}

Características clínicas. Se consignó edad, género, estado civil, nivel educacional, estado ocupacional, historia de tabaquismo y comorbilidades. La historia de tabaquismo incluyó la condición de fumador y el consumo de tabaco en paquetes/ año. La condición de ex-fumador se confirmó con cotinina urinaria menor a $10 \mathrm{mg} / \mathrm{L}$. Asimismo, se midió peso y talla, y se calculó el índice de masa corporal (IMC). La magnitud de la disnea se evaluó con la escala modificada del Medical Research Council (MMRC), que varía entre 0 y 4 puntos (Tabla 1) ${ }^{14}$.

Función pulmonar. Se efectuó una espirometría, se midieron los volúmenes pulmonares y la capacidad de difusión del monóxido de carbono (DLCO) según la normas internacionales ${ }^{15-17}$, empleando los valores de referencia de Hankinson et al ${ }^{18} y$ Quanjer et $\mathrm{al}^{16}$, respectivamente. La capacidad de ejercicio se evaluó con la prueba de caminata de 6 minutos $(\mathrm{PC} 6 \mathrm{M})^{19}$, midiendo la distancia recorrida (DR6) para los análisis.

Actividad física. La AF se evaluó con el Cuestionario Internacional de Actividad Física (IPAQ). Este cuestionario posee cuatro dominios relacionados con el tipo de actividad: a) Recreación; b) Doméstico y de jardinería; c) Trabajo, remunerado y no remunerado; y d) Transporte. Para cada dominio se diferencian tres tipos específicos de actividad: a) Caminata; b) Moderada intensidad; y c) Actividad vigorosa. La AF se describe en equivalentes metabólicos (METs), siendo un equivalente metabólico igual al gasto energético en reposo, y 
Tabla 1. Clasificación de la magnitud de la disnea según la escala modificada del Medical Research Council y de los niveles de actividad física según el Cuestionario Internacional de Actividad Física

\begin{tabular}{|c|c|}
\hline $\begin{array}{l}\text { Escala de disnea MMRC } \\
\text { Magnitud de la disnea }\end{array}$ & Descripción \\
\hline MMRC 0 & Disnea sólo con ejercicios extenuantes \\
\hline MMRC 1 & Disnea cuando camina rápido en plano o cuando sube una pendiente \\
\hline MMRC 2 & Debido a la disnea, camina más despacio en plano que sujetos de su edad \\
\hline MMRC 3 & $\begin{array}{l}\text { Debido a la disnea se detiene a descansar al caminar en plano unos } 100 \text { metros o después } \\
\text { de caminar algunos minutos }\end{array}$ \\
\hline MMRC 4 & Debido a la disnea no puede salir de la casa o bien requiere ayuda para vestirse o desvestirse \\
\hline $\begin{array}{l}\text { Cuestionario IPAQ } \\
\text { Nivel de actividad física }\end{array}$ & Criterio \\
\hline Baja & Sin actividad reportada o no incluido en los niveles moderado o alto \\
\hline Moderada & $\begin{array}{l}\text { Cualquiera de los siguientes: } \\
\text { a) Actividad intensa } \geq 3 \text { días, al menos } 20 \mathrm{~min} / \text { día } \\
\text { b) Actividad moderada y/ o caminata de al menos } 30 \mathrm{~min}, \geq 5 \text { días } \\
\text { c) Cualquier combinación de caminata, moderada o intensa, llegando a } 600 \text { METs-min/ } \\
\text { semana } \geq 5 \text { días }\end{array}$ \\
\hline Alta & $\begin{array}{l}\text { Cualquiera de los siguientes: } \\
\text { a) Actividad intensa al menos } 3 \text { días acumulando } 1.500 \mathrm{METS} \text {-min/ semana } \\
\text { b) Cualquier combinación de caminata, moderada o intensa, acumulando } 3.000 \mathrm{METs}-\mathrm{min} / \\
\text { semana } \geq 7 \text { días }\end{array}$ \\
\hline
\end{tabular}

Disnea MMRC: Magnitud de la disnea según la escala modificada del Medical Research Council; IPAQ: Cuestionario Internacional de Actividad Física (International Physical Activity Questionnaire).

se expresan en METs minutos/semana. El IPAQ considera actividades de intensidad moderada a aquellas de 3-6 METs y vigorosas a aquellas mayores de 6 METs y emplea los siguientes equivalentes metabólicos para cada nivel de actividad: caminar $=3,3$; actividad moderada $=4,0$; actividad vigorosa $=8,0$. Así, los METs min/semana corresponden al equivalente metabólico $\times$ minutos al día $\times$ días a la semana. El cuestionario clasifica adicionalmente la AF en tres niveles: baja, moderada y alta $(\text { Tabla } 1)^{9}$. La categoría alta estaría compuesta por individuos muy activos, mientras que la categoría baja incluiría a los sujetos sedentarios. Por último, el cuestionario incorpora un indicador adicional de actividad sedentaria, que no agrega puntaje, $y$ que corresponde al tiempo sentado, medido por separado para los días hábiles y los fines de semana.

\section{Análisis estadístico}

Los datos se expresan como promedios \pm 1 desviación estándar. La comparación entre controles y pacientes se realizó con la prueba de Mann-Whitney para muestras independientes. Para comparar la AF entre los controles y las 4 etapas de gravedad de GOLD se utilizó la prueba de Kruskal-Wallis, al igual que para comparar la $\mathrm{AF}$ de los controles y los pacientes, clasificados según la magnitud de la disnea MMRC. Las diferencias a posteriori entre los grupos se evaluaron con el método de Dunn. Para establecer las posibles relaciones entre la AF, la función pulmonar y la capacidad de ejercicio se empleó el coeficiente de correlación de Pearson o Spearman.

\section{Resultados}

\section{Características generales}

Las características sociodemográficas y funcionales del grupo control y los pacientes se describen en la Tabla 2. Los pacientes abarcaban todo el espectro de gravedad de la enfermedad según GOLD, tenían una edad algo superior, un leve predominio del sexo masculino, un nivel educacional inferior y un menor porcentaje de sujetos ocupacionalmente activos comparados con los controles. Las comorbilidades más frecuentes 
Tabla 2. Características sociodemográficas, antropométricas, de función pulmonar y capacidad de ejercicio en pacientes con EPOC y controles

\begin{tabular}{|c|c|c|c|}
\hline Características & $\begin{array}{c}\text { Controles } \\
n=55\end{array}$ & $\begin{array}{c}\text { EPOC } \\
n=112\end{array}$ & Valor-p \\
\hline Edad, años & $63 \pm 9$ & $66 \pm 6$ & 0,043 \\
\hline Sexo masculino & $23(41,8)$ & $67(59,8)$ & 0,03 \\
\hline $\mathrm{IMC}, \mathrm{kg} / \mathrm{m}^{2}$ & $27 \pm 4$ & $27 \pm 4$ & NS \\
\hline $\begin{array}{l}\text { Estado civil } \\
\text { Soltero/separado } \\
\text { Casado/pareja estable } \\
\text { Viudo }\end{array}$ & $\begin{array}{c}11(20) \\
40(72,7) \\
4(7,3)\end{array}$ & $\begin{array}{l}26(23,2) \\
69(61,6) \\
17(15,2)\end{array}$ & $\begin{array}{l}\text { NS } \\
\text { NS } \\
\text { NS }\end{array}$ \\
\hline $\begin{array}{l}\text { Educación } \\
\text { Básica o menor } \\
\text { Media incompleta } \\
\text { Media completa/Universitaria }\end{array}$ & $\begin{array}{c}3(5,5) \\
12(21,8) \\
40(72,7)\end{array}$ & $\begin{array}{c}33(29,5) \\
12(10,7) \\
67(59,8)\end{array}$ & $\begin{array}{c}<0,0001 \\
0,055 \\
\text { NS }\end{array}$ \\
\hline Ocupacionalmente activo & $27(49,1)$ & $30(27)$ & 0,004 \\
\hline $\begin{array}{l}\text { Tabaquismo } \\
\text { Historia, paquetes/año } \\
\text { Activo } \\
\text { Ex-fumador } \\
\text { Nunca fumador }\end{array}$ & $\begin{array}{l}14 \pm 20 \\
16(29,1) \\
12(21,8) \\
27(49,1)\end{array}$ & $\begin{array}{c}49 \pm 25 \\
32(28,6) \\
80(71,4) \\
0(0)\end{array}$ & $\begin{aligned}< & 0,0001 \\
& \text { NS } \\
< & 0,0001 \\
< & 0,0001\end{aligned}$ \\
\hline $\mathrm{VEF}_{1}, \%$ teórico & $107 \pm 15$ & $60 \pm 24$ & $<0,0001$ \\
\hline $\mathrm{PaO}_{2}, \mathrm{mmHg}$ & $82 \pm 8$ & $74 \pm 10$ & $<0,0001$ \\
\hline DLCO, \% teórico & $107 \pm 20$ & $84 \pm 26$ & $<0,0001$ \\
\hline $\mathrm{Cl} / \mathrm{CPT}, \%$ & $48 \pm 7$ & $34 \pm 10$ & $<0,0001$ \\
\hline DR6, metros & $572 \pm 74$ & $479 \pm 100$ & $<0,0001$ \\
\hline
\end{tabular}

Los valores se expresan como promedios \pm 1 desviación estándar o como número (porcentaje). IMC=índice de masa corporal; $E P O C=$ enfermedad pulmonar obstructiva crónica; $\mathrm{Cl}=$ capacidad inspiratoria; $\mathrm{CPT}=$ capacidad pulmonar total; DR6: distancia recorrida durante la prueba de caminata de 6 minutos. Comparaciones efectuadas con la prueba de Mann-Whitney para muestras independientes. fueron hipertensión arterial, sobre peso/obesidad, intolerancia a la glucosa/diabetes y depresión. La hipertensión arterial y la depresión resultaron más frecuentes en los pacientes ( 56 vs $33 \%, \mathrm{p}=0,004$; $\mathrm{y} 11$ vs $2 \%, p=0,044$, respectivamente).

\section{Actividad física en controles $y$ pacientes con EPOC}

En los pacientes, la AF total estaba significativamente reducida, mientras que el tiempo de permanencia sentado, tanto en días hábiles como durante los fines de semana, era significativamente mayor (Tabla 3). La menor AF se debió a una disminución de las actividades moderadas y de caminar, tanto domésticas como recreacionales (Figura 1). Como resultado, un mayor porcentaje de pacientes realizaba actividad sedentaria o baja, a diferencia de los controles, en que era mayor la proporción de individuos que efectuaba actividades moderadas o altas (Figura 2).

\section{Actividad física según la gravedad de la EPOC y la magnitud de la disnea MMRC}

La AF fue similar en el grupo control y en los pacientes GOLD 1, reduciéndose significativamente a

Tabla 3. Nivel de actividad física en pacientes con enfermedad pulmonar obstructiva crónica y controles

\begin{tabular}{|lccc|}
\hline $\begin{array}{l}\text { Actividad física IPAQ } \\
\text { Puntajes continuos }\end{array}$ & $\begin{array}{c}\text { Controles } \\
\mathbf{n = 5 5}\end{array}$ & $\begin{array}{c}\text { EPOC } \\
\mathbf{n = 1 1 2}\end{array}$ & Valor-p \\
\hline Actividad intensa, METs min/semana & $313 \pm 1.065$ & $192 \pm 769$ & NS \\
\hline Actividad moderada, METs min/semana & $1.710 \pm 2.069$ & $940 \pm 1.491$ & 0,003 \\
\hline Actividad leve, METs min/semana & $897 \pm 1.063$ & $691 \pm 1.448$ & 0,011 \\
\hline Total, METs min/semana & $2.920 \pm 3.040$ & $1.823 \pm 2.598$ & 0,001 \\
\hline Horas diarias sentado Lunes-Viernes & $6,06 \pm 2,9$ & $8,76 \pm 4,4$ & $<0,0001$ \\
\hline Horas diarias sentado Sábado-Domingo & $5,9 \pm 2,8$ & $8,5 \pm 4,3$ & $<0,0001$ \\
\hline
\end{tabular}

Los valores se expresan como promedios \pm 1 desviación estándar. EPOC $=$ enfermedad pulmonar obstructiva crónica. Las comparaciones se efectuaron con la prueba de Mann-Whitney para muestras independientes. MET corresponde a un equivalente metabólico $(3,5 \mathrm{ml} / \mathrm{kg} / \mathrm{min}$ o $1 \mathrm{kcal} / \mathrm{kg} / \mathrm{h})$. 

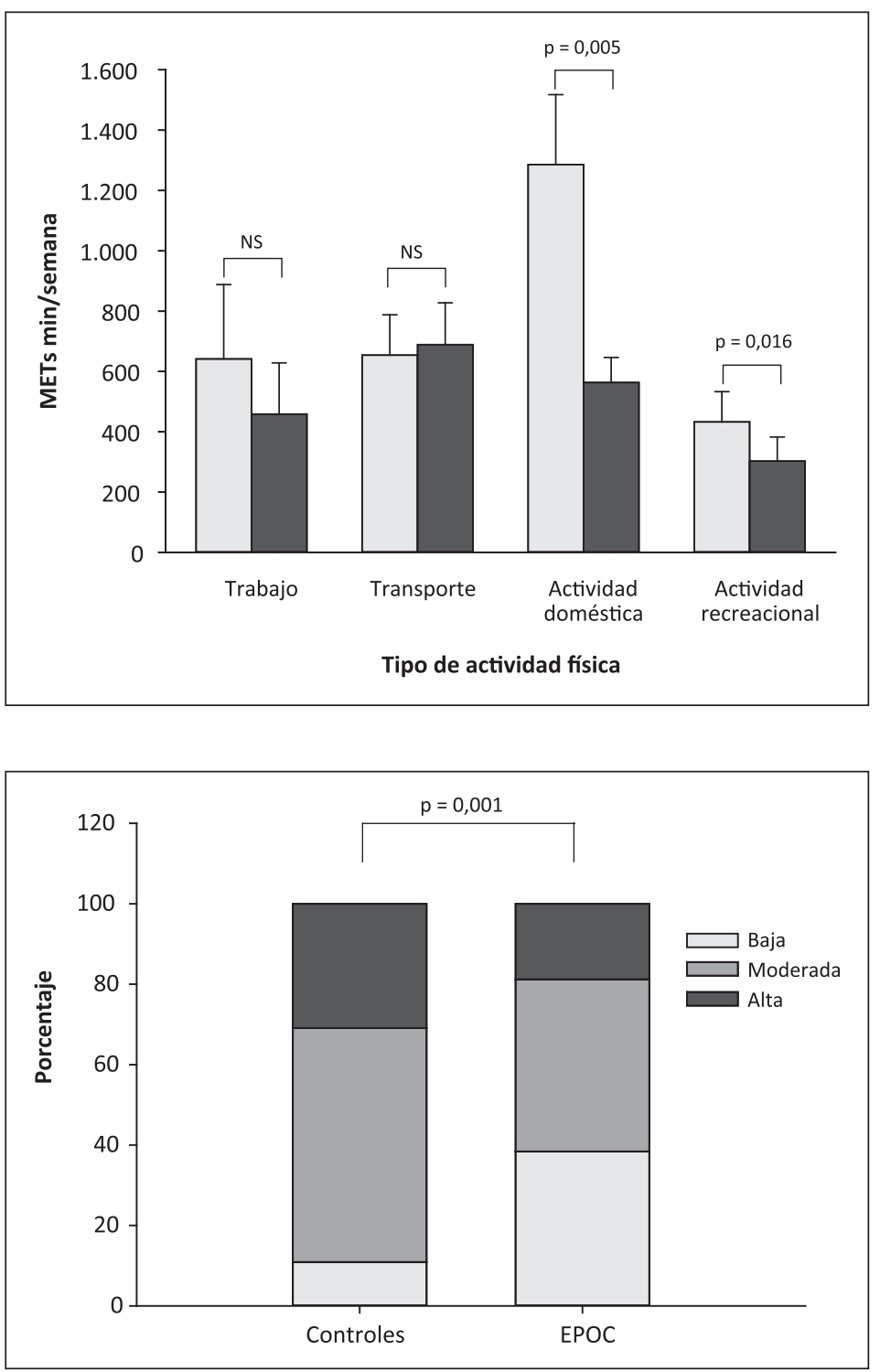

Figura 1. Magnitud de la actividad física según el tipo de actividad física, de acuerdo al Cuestionario Internacional de Actividad Física en 55 controles y 112 pacientes con EPOC. Los datos se expresan como promedios $\pm 1 \mathrm{EE}$. Las barras grises representan a los controles y las negras, a los pacientes. Las diferencias entre controles y pacientes se evaluaron con la prueba de Mann-Whitney para muestras independientes.

Figura 2. Intensidad de la actividad física según el Cuestionario Internacional de Actividad Física en 55 controles y 112 pacientes con enfermedad pulmonar obstructiva crónica. Las barras apiladas representan el porcentaje de sujetos de cada grupo cuya actividad es clasificada como leve, moderada o alta.

partir de la etapa GOLD 2 (Figura 3; panel superior). En concordancia con ello, la intensidad de la AF disminuyó desde la etapa GOLD 2 en adelante, reduciéndose a la mitad el porcentaje de pacientes clasificados como muy activos y aumentando a más del doble el porcentaje de individuos sedentarios (Figura 3; panel inferior).

La AF no mostró diferencias entre el grupo control y los pacientes sin disnea $(\mathrm{MMRC}=0)$, pero se redujo progresivamente a partir de un puntaje de disnea MMRC=1 (Figura 4; panel superior). A su vez, mientras el porcentaje de pacientes muy activos disminuyó desde el puntaje $\mathrm{MMRC}=1$, también aumentó notoriamente la proporción de pacientes sedentarios (Figura 4, panel inferior).

\section{Asociaciones entre actividad física, variables socio-demográficas, función pulmonary capacidad de ejercicio}

En el grupo total, la AF no varió según el género, estado civil, ni la condición de fumador. Tampoco influyó la presencia de obesidad y no se observó una correlación significativa con el IMC. En cambio, fue inferior en los sujetos sin 


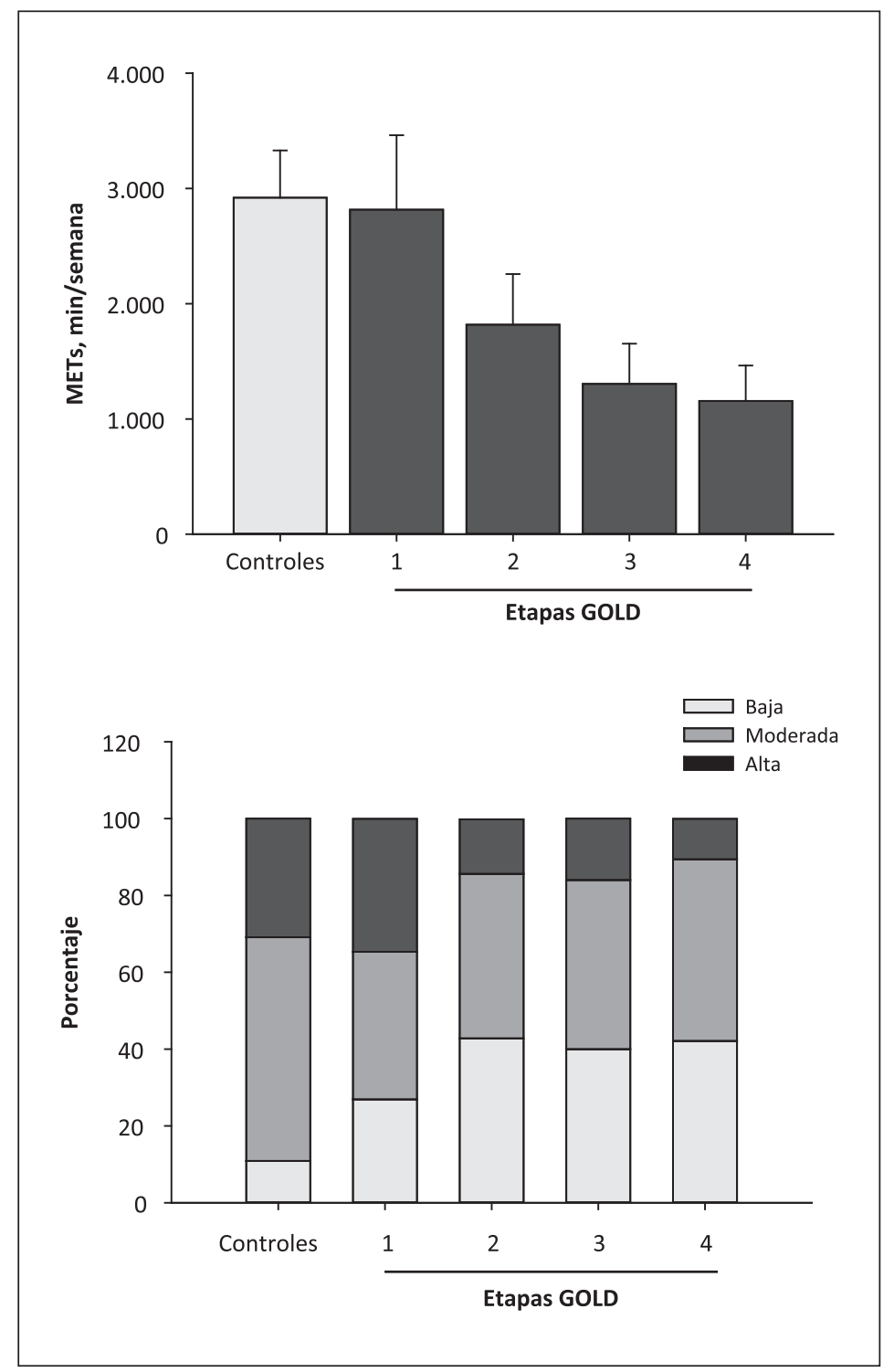

Figura 3. Panel superior: Actividad física según el Cuestionario Internacional de Actividad Física en el grupo de controles y pacientes con EPOC, clasificados según los criterios de gravedad de GOLD. Los datos se expresan como promedios $\pm 1 \mathrm{EE}$. La actividad física de las etapas GOLD 2, 3 y 4 es significativamente menor que la de los controles $(p=0,002 ; p=0,001$; y $p=0,003$ ， respectivamente); la de las etapas GOLD 2 , 3 y 4 es significativamente inferior a la de GOLD 1 ( $p=0.034 ; p=0,017 ; p=0,025$, respectivamente). Panel inferior: Intensidad de la actividad física según el Cuestionario Internacional de Actividad Física en ambos grupos. Las barras apiladas representan el porcentaje de sujetos de cada grupo cuya actividad es clasificada como leve, moderada o alta. Chi-cuadrado; $p=0,013$. EPOC: Enfermedad pulmonar obstructiva crónica; GOLD: Global strategy for the diagnosis, management, and prevention of COPD. ocupación $(\mathrm{n}=110 ; \mathrm{p}=0,028)$ y en aquellos con depresión diagnosticada $(\mathrm{n}=13 ; \mathrm{p}=0,047)$; a la vez que presentó una correlación inversa con la edad $(r=-0,21 ; p=0,004)$. La Tabla 4 describe las correlaciones significativas, aunque bajas, con el $\mathrm{VEF}_{1}$, la DLCO, el índice capacidad inspiratoria/ capacidad pulmonar total y la DR6.

\section{Discusión}

El presente estudio describe la actividad física en un número sustancial de pacientes con EPOC, concluyendo que en comparación con controles de edad semejante: a) es inferior y de menor intensidad; b) se hace evidente precozmente, desde la etapa GOLD 2 y desde un puntaje de disnea $\mathrm{MMRC}=1 ; \mathrm{y}, \mathrm{c})$ no se correlaciona estrechamente con la función pulmonar ni con la capacidad de ejercicio.

$\mathrm{Al}$ igual que en el presente estudio, pero empleando cuestionarios diferentes, otros autores han observado una reducción significativa de la $\mathrm{AF}$ total en los pacientes con EPOC en comparación con controles de edad similar ${ }^{20-23}$. Una ventaja de 


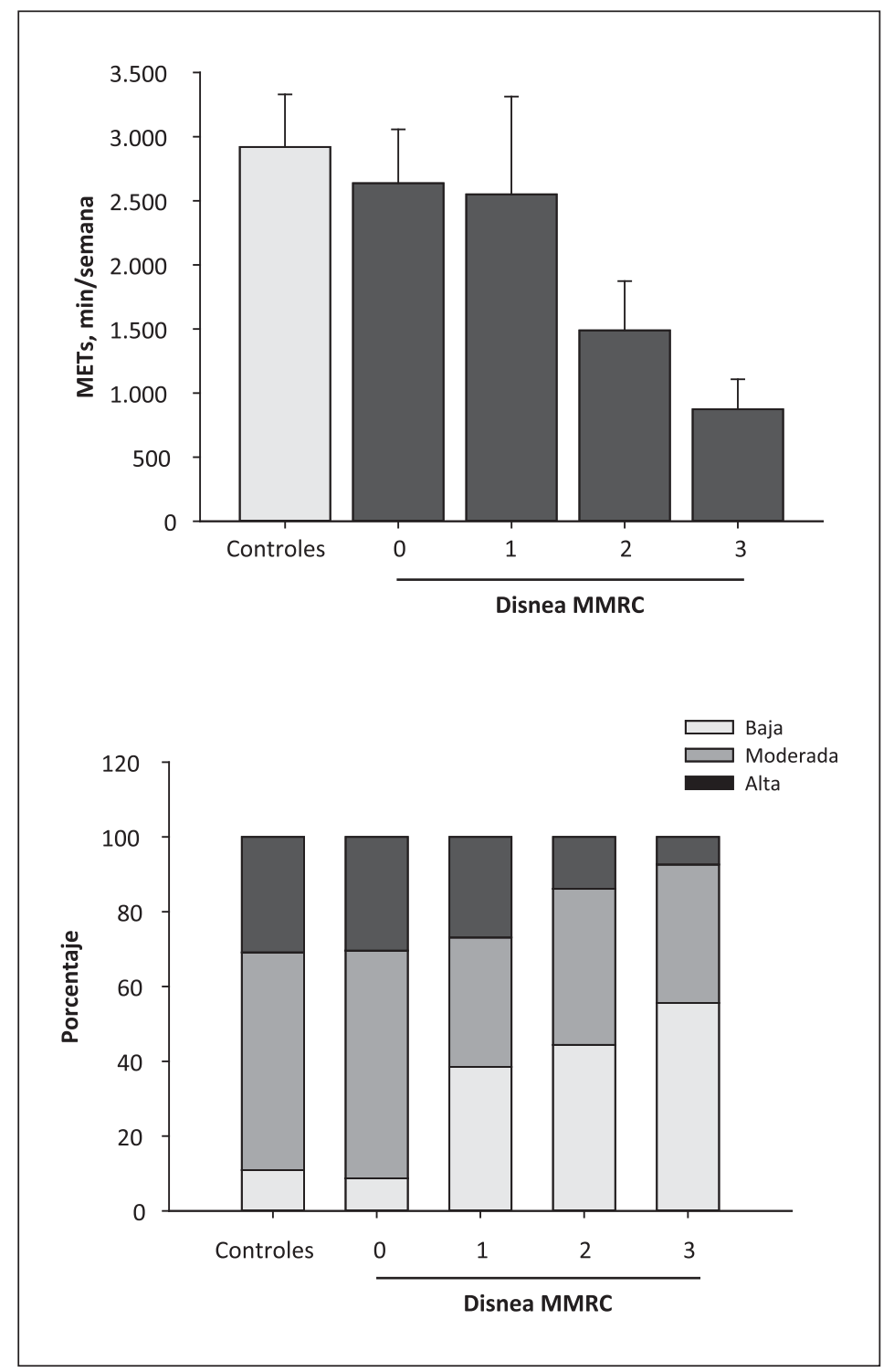

Figura 4. Panel superior: Actividad física según el Cuestionario Internacional de Actividad Física en controles y pacientes con EPOC, clasificados según la magnitud de la disnea con la escala MMRC. Los datos se expresan como promedios $\pm 1 \mathrm{EE}$. La actividad física de los pacientes con puntaje de disnea MMRC 1, 2 y 3 es significativamente inferior a la de los controles $(p=0,05 ; p=0,001$; $p<0,0001$, respectivamente). La actividad física de los pacientes con disnea MMRC 1 , 2 y 3 es significativamente inferior a la de los pacientes sin disnea, esto es, MMRC = $0(p=0,043 ; p=0,002 ; y p<0,0001$, respectivamente). La actividad física de los pacientes con disnea MMRC $=3$ es significativamente menor a la de aquellos con disnea $M M R C=1$. Panel inferior: Intensidad de la actividad física según el Cuestionario Internacional de Actividad Física en ambos grupos. Las barras apiladas representan el porcentaje de sujetos de cada grupo cuya actividad es clasificada como leve, moderada o alta. $\chi^{2} ; p<0,0001$. Disnea MMRC: escala de disnea modificada del Medical Research Council. nuestro estudio sobre aquellos es el mayor número de pacientes incluido, que asimismo abarca todo el espectro de gravedad de la enfermedad.

La menor AF de los pacientes con EPOC ha sido confirmada además mediante podómetros y acelerómetros ${ }^{24-27}$. Según estos estudios, la AF diaria de los pacientes representaría apenas 57\% de la actividad del grupo control $^{28}$, porcentaje muy similar al observado en este estudio, en que la AF semanal de los pacientes representó $62 \%$ de la actividad realizada por los controles (Tabla 3 ).

Según nuestros datos, la intensidad de la $\mathrm{AF}$ de los pacientes fue, a su vez, significativamente inferior a la de los controles. Sólo 43\% de los pacientes era moderadamente activo y apenas un $19 \%$, muy activo, en contraste con 58 y $31 \%$ de los controles, respectivamente (Figura 1). Por el contrario, $38 \%$ de los pacientes fue calificado como sedentario, en comparación con $11 \%$ de los controles. Se ha descrito que, en general, la intensidad de la AF de los pacientes con EPOC no supera el $75 \%$ de aquella observada en los controles ${ }^{28}$, lo que nuevamente es concordante con nuestros resultados. La frecuencia de sedentarismo entre 


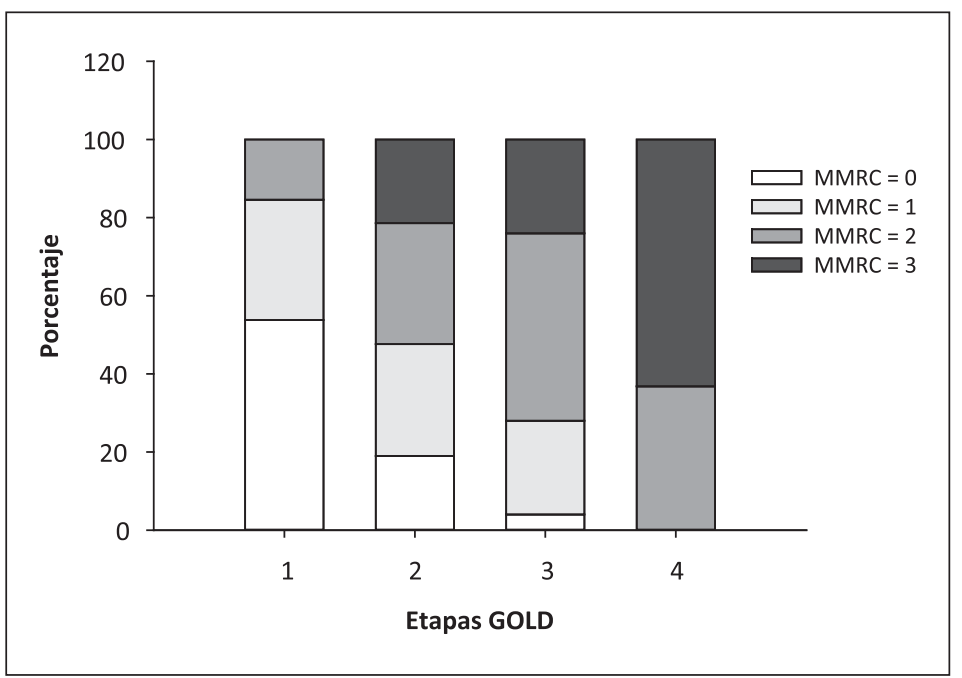

Figura 5. Magnitud de la disnea en 112 pacientes con enfermedad pulmonar obstructiva crónica, clasificados según los criterios de gravedad de GOLD. Los datos se presentan como barras apiladas y representan el porcentaje de sujetos de cada etapa GOLD cuya disnea es clasificada en los diferentes puntajes de la escala de disnea MMRC. Nótese que no se incluyeron pacientes con puntaje MMRC $=4$. GOLD: Global strategy for the diagnosis, management, and prevention of COPD. Disnea MMRC: escala de disnea modificada del Medical Research Council.
Tabla 4. Correlaciones entre la actividad física y variables antropométricas, de función pulmonar y capacidad de ejercicio en $\mathbf{1 1 2}$ pacientes con enfermedad pulmonar obstructiva crónica

\begin{tabular}{|lcc|}
\hline Variables & $\begin{array}{c}\text { METs min/semana } \\
\text { totales }\end{array}$ & Valor-p \\
\hline $\mathrm{IMC}_{1} \mathrm{~kg} / \mathrm{m}^{2}$ & $-0,11^{*}$ & $\mathrm{NS}$ \\
$\mathrm{VEF}_{1}, \%$ teórico & $0,31^{*}$ & 0,001 \\
$\mathrm{CVF}_{,} \%$ teórico & $0,18^{*}$ & $\mathrm{NS}$ \\
$\mathrm{PaO}_{2}, \mathrm{mmHg}$ & $0,16^{*}$ & $\mathrm{NS}$ \\
\hline $\mathrm{DLCO}, \%$ teórico & $0,32^{*}$ & 0,001 \\
\hline $\mathrm{Cl} / \mathrm{CPT} \%$ & $0,27^{*}$ & 0,004 \\
\hline DR6, metros & $0,29^{*}$ & 0,002 \\
\hline Etapas GOLD* & $-0,23^{\dagger}$ & 0,016 \\
\hline Disnea MMRC* & $-0,39^{\dagger}$ & $<0,0001$ \\
\hline
\end{tabular}

IMC: índice de masa corporal; $\mathrm{VEF}_{1}$ : volumen espiratorio forzado del primer segundo; DLCO: capacidad de difusión; Cl: capacidad inspiratoria; CPT: capacidad pulmonar total; DR6: distancia recorrida en 6 minutos durante la prueba de caminata de 6 minutos; GOLD: Global strategy for the diagnosis, management, and prevention of COPD; Disnea MMRC: magnitud de la disnea según la escala modificada del Medical Research Council. *Correlación $r$ de Pearson. †Correlación rho de Spearman.

los controles, en cambio, resultó notoriamente inferior a la obtenida en el mismo tramo de edad en la Encuesta Nacional de Salud 2009-2010 (27\% entre 45-64 años y $49 \%$ sobre 65 años ${ }^{29}$. Si bien en la Encuesta Nacional de Salud (ENS) se empleó un cuestionario diferente (Cuestionario de Actividad
Física Global; GPAQ), se ha demostrado que éste es comparable al Cuestionario IPAQ ${ }^{30}$, por lo que pensamos que las diferencias podrían deberse a un sesgo de selección. No podemos descartar, en efecto, que más sujetos físicamente activos hayan aceptado participar como controles, los que a su vez exhibieron una menor edad (Tabla 2) y un leve predominio de individuos $<65$ años en comparación con los pacientes ( 55 vs $46 \%$ ). Sin embargo, este sesgo a favor de una mayor AF del grupo control es aminorado en parte por la mayor proporción de hombres entre los pacientes, pues los hombres demostraron mayores niveles de AF que las mujeres en la ENS. Independientemente de las consideraciones anteriores, en el presente estudio observamos además que los pacientes con EPOC permanecían sentados un tiempo significativamente mayor que los controles, apoyando el sedentarismo que caracterizaría a esta población, y que es coincidente con dos comunicaciones previas, en las cuales se emplearon acelerómetros ${ }^{31,32}$.

El efecto de la gravedad de la EPOC y la magnitud de la disnea sobre la AF sólo ha sido evaluado previamente por Watz et $\mathrm{al}^{11}$ empleando acelerómetros. Es interesante destacar que ambos estudios coinciden en señalar que la AF se reduce desde de la etapa GOLD 2 y con un puntaje de disnea $\mathrm{MMRC}=1$. Más importante aún, en ambos estudios la etapa GOLD 2 y la magnitud de la disnea $\mathrm{MMRC}=1$ marcan un punto de inflexión en que la proporción de sujetos sedentarios aumenta notoriamente. Así, en nuestro estudio entre la eta- 
pa GOLD 1 y 2, la proporción de pacientes muy inactivos aumentó de 27 a 43\%, manteniéndose en un porcentaje similar hasta la etapa GOLD 4.El efecto de la disnea es más marcado, pues en la transición entre los pacientes sin disnea $(\mathrm{MMRC}=0)$ y aquellos con puntaje MMRC $=1$, los individuos sedentarios aumentaron de 9 a 39\%, y esta proporción creció a $56 \%$ con un puntaje $\mathrm{MMRC}=3$.

Aunque nuestros datos indican que la inactividad en la EPOC es frecuente y que se debería indagar prácticamente en todo paciente con disnea leve, no proporcionan información confiable que permita identificar al sujeto sedentario en la práctica clínica diaria. En el caso particular de las etapas GOLD y la disnea (MMRC), la dificultad se explica porque en cada etapa GOLD la magnitud de la disnea es muy variable, como lo demuestra la Figura 5, y como ha sido recientemente destacado por otros ${ }^{33}$. Además, la AF no sólo depende de alguna característica fisiológica particular, sino también de aspectos psicosociales y ambientales (estado ocupacional, estado civil, presencia de depresión, entre otros). En nuestro estudio, la presencia de depresión y un bajo nivel educacional, además de la inactividad laboral, fueron más frecuentes entre los pacientes, concordando con hallazgos previamente descritos ${ }^{34,35}$ e influyeron negativamente sobre la actividad física. Así se explican, en parte, las débiles asociaciones entre la AF y distintas variables funcionales descritas en la Tabla 5.

Aunque la escasa correlación entre la AF y la PC6M podría parecer contradictoria a primera vista, se debe simplemente a que suele confundirse capacidad de ejercicio, que es lo que mide la PC6M, con el ejercicio efectivamente realizado, que es la AF propiamente tal. Estos datos concuerdan con los de Watz et $\mathrm{al}^{11} \mathrm{y}$ son sustentados por Pitta et $\mathrm{al}^{32}$, quienes demostraron que la PC6M es un pobre predictor de la AF, particularmente cuando la distancia recorrida promedio supera los $400 \mathrm{~m}$, como ocurre en el presente estudio.

Nuestro estudio posee algunas limitaciones que merecen mencionarse. Aunque el IPAQ ha sido evaluado internacionalmente en sujetos sanos, no existe una validación formal en pacientes con EPOC. En aquellos estudios realizados en población normal se ha observado que el instrumento posee una adecuada consistencia interna, una buena reproducibilidad ${ }^{36,37}$ y una moderada concordancia con acelerómetros, particularmen- te para la caminata y las actividades vigorosas ${ }^{37}$. En nuestra opinión, la que es compartida por otros autores ${ }^{10}$, ningún instrumento, subjetivo u objetivo, ha demostrado aún su superioridad. De hecho, recientemente se ha cuestionado la capacidad de los acelerómetros para determinar la validez de los cuestionarios, pues los puntos de corte para definir la intensidad de las actividades varían entre ambos instrumentos ${ }^{38}$. Esto podría explicar, por ejemplo, que exista una muy buena concordancia entre dos cuestionarios diferentes y entre dos tipos de acelerómetros ${ }^{38}$, pero no entre los cuestionarios y los acelerómetros ${ }^{37,39}$. Aun así, cabe una nota de cautela respecto a la exactitud de nuestras mediciones en pacientes con EPOC, ya que estos suelen sobrestimar su nivel de AF al emplear cuestionarios ${ }^{40}$, aun cuando se empleen las recomendaciones propuestas por el IPAQ para evitar este problema ${ }^{41}$.

En resumen, nuestros resultados indican que en pacientes con EPOC que representan todo el espectro de la enfermedad, la actividad física está reducida en magnitud e intensidad al compararla con controles de edad semejante. Esta reducción de la actividad física es más evidente a partir de la EPOC moderada (GOLD 2) y cuando ya existe disnea leve según la escala MMRC. Consecuentemente, la rehabilitación respiratoria debería constituirse en un objetivo muy precoz en el tratamiento de esta enfermedad.

\section{Referencias}

1. Thompson PD, Buchner D, Pina IL, Balady GJ, Williams MA, Marcus BH, et al. Exercise and physical activity in the prevention and treatment of atherosclerotic cardiovascular disease: A statement from the Council on Clinical Cardiology (Subcommittee on Exercise, Rehabilitation, and Prevention) and the Council on Nutrition, Physical Activity, and Metabolism (Subcommittee on Physical Activity). Circulation 2003; 107: 3109-16.

2. García-Aymerich J, Lange P, Benet M, Schnohr P, Anto JM. Regular physical activity modifies smoking-related lung function decline and reduces risk of chronic obstructive pulmonary disease: A population-based cohort study. Am J Respir Crit Care Med 2007; 175: 458-63.

3. García-Aymerich J, Farrero E, Félez MA, Izquierdo J, Marrades RM, Antó JM. Risk factors of readmission to hospital for a COPD exacerbation: a prospective study. Thorax 2003; 58: 100-5. 
4. García-Aymerich J, Lange P, Benet M, Schnohr P, Antó JM. Regular physical activity reduces hospital admission and mortality in chronic obstructive pulmonary disease: a population based cohort study. Thorax 2006; 61: 7728.

5. Pitta F, Troosters T, Probst VS, Spruit MA, Decramer M, Gosselink R. Quantifying physical activity in daily life with questionnaires and motion sensors in COPD. Eur Respir J 2006; 27: 1040-55.

6. Walker PP, Burnett A, Flavahan PW, Calverley PMA. Lower limb activity and its determinants in COPD. Thorax 2008; 63: 683-9.

7. García-Aymerich J, Serra I, Gómez FP, Farrero E, Balcells E, Rodríguez DA, et al. Physical activity and clinical and functional status in COPD. Chest 2009; 136: 62-70.

8. Guirao-Goris JA, Cabrero-García J, Moreno Pina JP, Muñoz-Mendoza CL. Revisión estructurada de los cuestionarios y escalas que miden la actividad física en los adultos mayores y ancianos. Gac Sanit 2009; 23: 334. e351-334.e367.

9. Craig CL, Marshall AL, Sjostrom M, Bauman AE, Booth $\mathrm{ML}$, Ainsworth BE, et al. International physical activity questionnaire: 12-country reliability and validity. Med Sci Sports Exerc 2003; 35: 1381-95.

10. Roche N. Activity limitation: a major consequence of dyspnoea in COPD. Eur Respir Rev 2009; 18: 54-7.

11. Watz H, Waschki B, Meyer T, Magnussen H. Physical activity in patients with COPD. Eur Respir J 2009; 33: 262-72.

12. Rabe KF, Hurd S, Anzueto A, Barnes PJ, Buist SA, Calverley $\mathrm{P}$, et al. Global strategy for the diagnosis, management, and prevention of chronic obstructive pulmonary disease: GOLD executive summary. Am J Respir Crit Care Med 2007; 176: 532-55.

13. McCormack GR, Friedenreich C, Shiell A, Giles-Corti B, Doyle-Baker PK. Sex- and age-specific seasonal variations in physical activity among adults. J Epidemiol Community Health 2010; 64: 1010-6.

14. Mahler DA, Wells CK. Evaluation of clinical methods for rating dyspnea. Chest 1988; 93: 580-6.

15. Miller MR, Hankinson J, Brusasco V, Burgos F, Casaburi $\mathrm{R}$, Coates A, et al. Standardisation of spirometry. Eur Respir J 2005; 26: 319-38.

16. Quanjer P, Tammeling G, Cotes J, Pedersen O, Peslin R, Yernault J. Lung volumes and forced ventilatory flows. Report work party: Standardization of lung function testing. Eur Respir J 1993; 6 Suppl. 16: 5s-40s.

17. MacIntyre N, Crapo RO, Viegi G, Johnson DC, van der Grinten CPM, Brusasco V, et al. Standardisation of the single-breath determination of carbon monoxide uptake in the lung. Eur Respir J 2005; 26: 720-35.
18. Hankinson J, Odencrantz J, Fedan K. Spirometric reference values from a sample of the general U.S. population. Am J Respir Crit Care Med 1999; 159: 179-87.

19. ATS Statement: Guidelines for the six-minute walk test. Am J Respir Crit Care Med 2002; 166: 111-7.

20. Garrod R, Bestall JC, Paul EA, Wedzicha JA, Jones PW. Development and validation of a standardized measure of activity of daily living in patients with severe COPD: the London Chest Activity of Daily Living scale (LCADL). Respir Med 2000; 94: 589-96.

21. Miravitlles M, Soriano JB, García-Río F, Muñoz L, Duran-Tauleria E, Sánchez G, et al. Prevalence of COPD in Spain: impact of undiagnosed COPD on quality of life and daily life activities. Thorax 2009; 64: 863-8.

22. Inal-Ince D, Savci S, Coplu L, Arikan H. Factors determining self-efficacy in chronic obstructive pulmonary disease. Saudi Med J 2005; 26: 542-7.

23. Gosker HR, Lencer NHMK, Franssen FME, van der Vusse GJ, Wouters EFM, Schols AMWJ. Striking similarities in systemic factors contributing to decreased exercise capacity in patients with severe chronic heart failure or COPD. Chest 2003; 123: 1416-24.

24. Schonhofer B, Ardes P, Geibel M, Kohler D, Jones P. Evaluation of a movement detector to measure daily activity in patients with chronic lung disease. Eur Respir J 1997; 10: 2814-9.

25. Troosters T, Sciurba F, Battaglia S, Langer D, Valluri SR, Martino L et al. Physical inactivity in patients with COPD, a controlled multi-center pilot-study. Respir Med 2010; 104: 1005-11.

26. Lores V, García-Ríoa F, Rojo B, Alcolea S, Mediano O. [Recording the daily physical activity of COPD patients with an accelerometer: An analysis of agreement and repeatability]. Arch Bronconeumol 2003; 42: 627-32.

27. Sandland C, Singh S, Curcio A, Jones P, Morgan M. A profile of daily activity in chronic obstructive pulmonary disease. J Cardiopulm Rehabil 2005; 25: 181-3.

28. Vorrink S, Kort H, Troosters T, Lammers J-W. Level of daily physical activity in individuals with COPD compared with healthy controls. Respir Res 2011; 12: 33.

29. Encuesta Nacional de Salud-2009: Ministerio de Salud Chile. Departamento de Epidemiología http://www. encuestasalud.cl/

30. Bull FC, Maslin T, Armstrong T. Global Physical Activity Questionnaire (GPAQ): Nine country reliability and validity study J Phys Act Health 2009; 6: 790-804.

31. Hernandes NA, Teixeira DdC, Probst VS, Brunetto AF, Ramos EMC, Pitta F. Profile of the level of physical activity in the daily lives of patients with COPD in Brazil. J Bras Pneumol 2009; 35: 949-56.

32. Pitta F, Troosters T, Spruit MA, Probst VS, Decramer 
M, Gosselink R. Characteristics of physical activities in daily life in chronic obstructive pulmonary disease. Am J Respir Crit Care Med 2005; 171: 972-7.

33. Agusti A, Calverley P, Celli B, Coxson H, Edwards L, Lomas D, et al. Characterisation of COPD heterogeneity in the ECLIPSE cohort. Respir Res 2010; 11: 122.

34. Maurer J, Rebbapragada V, Borson S, Goldstein R, Kunik ME, Yohannes AM et al. Anxiety and depression in COPD. Chest 2008; 134: 43S-56S.

35. Prescott E, Lange P, Vestbo J. Socioeconomic status, lung function and admission to hospital for COPD: results from the Copenhagen City Heart Study. Eur Respir J 1999; 13: 1109-14.

36. Brown WJ, Trost SG, Bauman A, Mummery K, Owen N. Test-retest reliability of four physical activity measures used in population surveys. J Sci Med Sport 2004; 7: 205-15.

37. Kurtze N, Rangul V, Hustvedt B-E. Reliability and validity of the international physical activity questionnaire in the Nord-Trondelag health study (HUNT) population of men. BMC Med Res Methodol 2008; 8: 63.

38. Macfarlane DJ, Lee CCY, Ho EYK, Chan KL, Chan D. Convergent validity of six methods to assess physical activity in daily life. J Appl Physiol 2006; 101: 1328-34.

39. Boon RM, Hamlin MJ, Steel GD, Ross JJ. Validation of the New Zealand Physical Activity Questionnaire (NZPAQ-LF) and the International Physical Activity Questionnaire (IPAQ-LF) with accelerometry. Br J Sports Med 2010; 44: 741-6.

40. Pitta F, Troosters T, Spruit MA, Decramer M, Gosselink R. Activity monitoring for assessment of physical activities in daily life in patients with chronic obstructive pulmonary disease. Arch Phys Med Rehabil 2005; 86: 1979-85.

41. Rzewnicki R, Auweele YV, Bourdeaudhuij ID. Addressing overreporting on the International Physical Activity Questionnaire (IPAQ) telephone survey with a population sample. Public Health Nutr 2003; 6: 299-305. 